\title{
THE DESIGN OF 1 N•M TORQUE STANDARD MACHINE AT NIM
}

\author{
ZHIMIN ZHANG \\ Mechanics and Acoustics Division, National Institute of Metrology, \\ No.18, Bei San Huan Dong Lu, Beijing, 100013, China \\ zhiminzhang@nim.ac.cn \\ YUE ZHANG \\ Mechanics and Acoustics Division, National Institute of Metrology, \\ No.18, Bei San Huan Dong Lu, Beijing, 100013, China \\ zhangy@nim.ac.cn \\ TAO LI \\ Torque Laboratory, Shang Hai Marine Equipment Research Institute, \\ No.10, Heng Shan Road, Shanghai, 130025, China \\ litao117@yahoo.com.cn \\ HONGLEI JI \\ Torque Laboratory, Shang Hai Marine Equipment Research Institute, \\ No.10, Heng Shan Road, Shanghai, 130025, China \\ thronejhl@yahoo.com.cn
}

\begin{abstract}
A $1 \mathrm{~N} \cdot \mathrm{m}$ torque standard machine has been designed and developed at National Institute of Metrology (NIM) since 2011. The torque standard machine adopts the moment arm-deadweight type, the air bearing with low friction is adopted to support the moment-arm at the fulcrum, the invar with the low expansion coefficient is used as the material of the moment-arm, the weight suspension part and weight loading system are specially designed to ensure the applied force by small weights accurately and reliably. This paper introduces the mechanical structures of the machine, the electrical control system is described. $1 \mathrm{~N} \bullet \mathrm{m}$ torque standard machine is capable of realizing the torque from $1 \mathrm{mN} \bullet \mathrm{m}$ to $1 \mathrm{~N} \bullet \mathrm{m}$ both in clockwise and anti-clockwise directions.
\end{abstract}

Keywords: Torque standard machine; air bearing; moment-arm; weight loading system.

\section{Introduction}

Torque measurement is an essential element on the development, quality inspection, safety and optimal control of mechanical products. Torque, as a basic parameter, is widely used in automobile, communications, aerospace, shipping and other fields. In

This is an Open Access article published by World Scientific Publishing Company. It is distributed under the terms of the Creative Commons Attribution 3.0 (CC-BY) License. Further distribution of this work is permitted, provided the original work is properly cited. 
recent year with the development of the electronics, automobile, aerospace and medical industry, there is a growing demand for the small torque measurement. In the aerospace and automobile sectors, small motor has a wide range of applications, accurate torque measurement of small motor is essential to ensure the product quality. Slight torque tightening tools are widely used in mobile phones, hard drives, cameras, laptop computers, automobile electronics, medical devices and other assembly industries, by using the tightening tools with torque sensor, precise torque control is achieved and product quality is improved. The establishment of small torque standard machine may provide reliable technical support for various industries.

National metrology institute is responsible for establishing and maintaining national torque standards, ensuring accuracy and consistency of torque dissemination and providing calibration services for customers in various sectors of industry. In the past years 4 sets of torque standard machine have been established at NIM, including $50 \mathrm{~N} \bullet \mathrm{m}$, $1 \mathrm{kN} \bullet \mathrm{m}, 5 \mathrm{kN} \bullet \mathrm{m}$ torque standard machines with the knife-edge support and $100 \mathrm{~N} \bullet \mathrm{m}$ torque standard machine with the air bearing support, these torque standard machines are capable of realizing the torque in the range of $0.5 \mathrm{~N} \cdot \mathrm{m}-5 \mathrm{kN} \bullet \mathrm{m}$. In order to meet the requirement of small torque measurement, a $1 \mathrm{~N} \bullet \mathrm{m}$ torque standard machine has been developed at NIM since 2011, the range of the small torque machine is from $1 \mathrm{mN} \bullet \mathrm{m}$ to $1 \mathrm{~N} \bullet \mathrm{m}$. The machine will be accomplished in the end of 2013.

\section{The Mechanical Structure of $1 \mathbf{N} \bullet \mathbf{m}$ Torque Standard Machine}

$1 \mathrm{~N} \bullet \mathrm{m}$ torque standard machine adopts the moment arm-deadweight type. The machine consists mainly of air bearing, moment-arm part, weight suspension part, weights loading system, transducer couplings, counter bearing part and pedestal part. The mechanical structure of $1 \mathrm{~N} \bullet \mathrm{m}$ torque machine is shown in Fig. 1. 


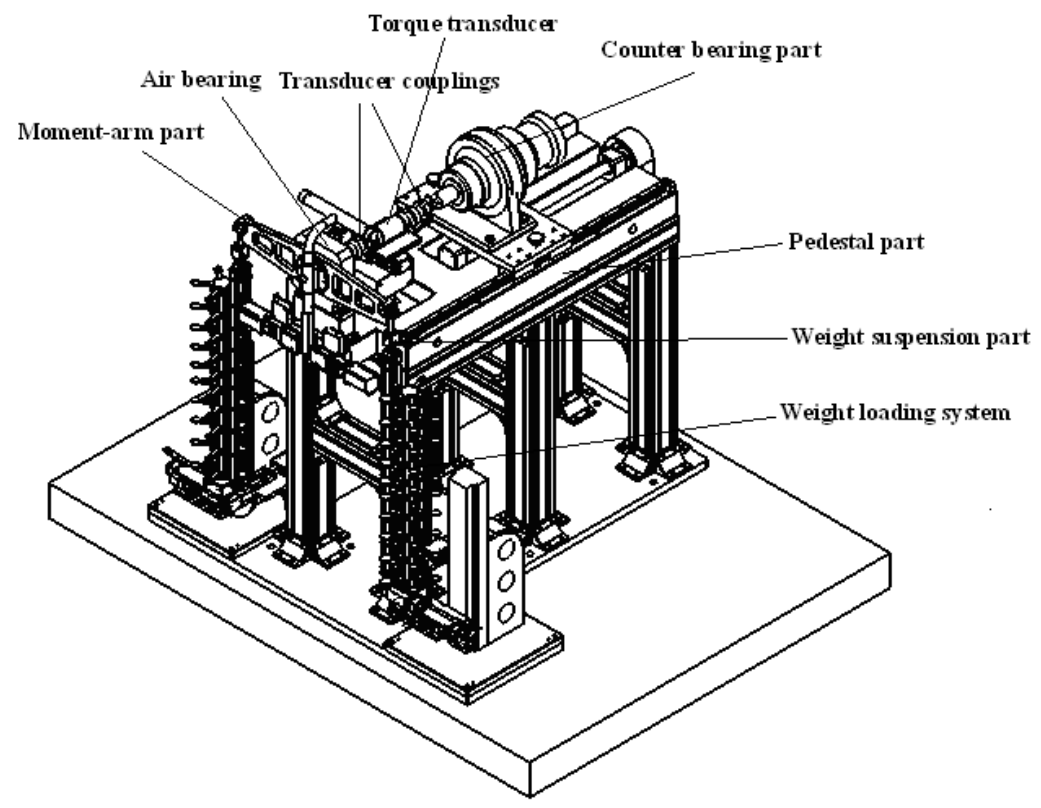

Fig.1. Mechanical structure of $1 \mathrm{~N} \bullet \mathrm{m}$ torque standard machine.

\subsection{Air bearing}

The X-type air bearing is adopted as the moment-arm support to minimize the friction at the fulcrum. Fig. 2 shows the structure of X-type air bearing. The work pressure of compressed air supplied to the air bearing is 3 bar. The mist separator with membrane air dryer and fine filters are used to eliminate the dust and moisture from the compressed air. The residual friction of air bearing is $0.3 \mu \mathrm{N} \bullet \mathrm{m}$.

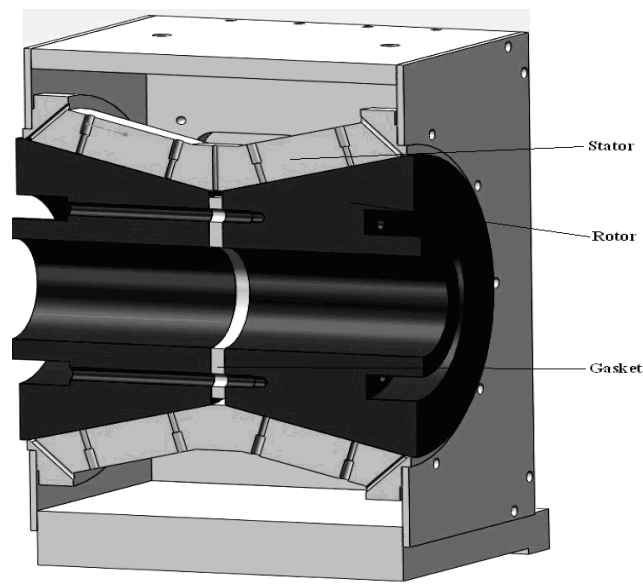

Fig.2. Structure of X-type air bearing. 


\subsection{Moment-arm system}

A single beam structure is adopted as the moment-arm, the weight loss holes are designed to reduce the weight of moment-arm. The balance beam is equipped so that the mass center of moment-arm may be adjusted by regulating two hand wheels in two ends of balance beam. Overload protection part is used to control the swing amplitude of the moment-arm and to ensure the moment-arm in the normal work status. The laser displacement sensors are used to measure the position of moment-arm, and the momentarm is adjusted to the initial horizontal position by controlling the counter bearing drive. The nominal length of the moment-arm is $250 \mathrm{~mm}$. The invar with the low thermal expansion coefficient is used as the materials of the moment-arm in order to reduce the uncertainty caused by length change of moment-arm due to the temperature variation.

The weight suspension assembly is designed to keep the force applied by small weights vertically downward and be free from the parasitic force. The structure of the moment-arm is shown in Fig. 3.

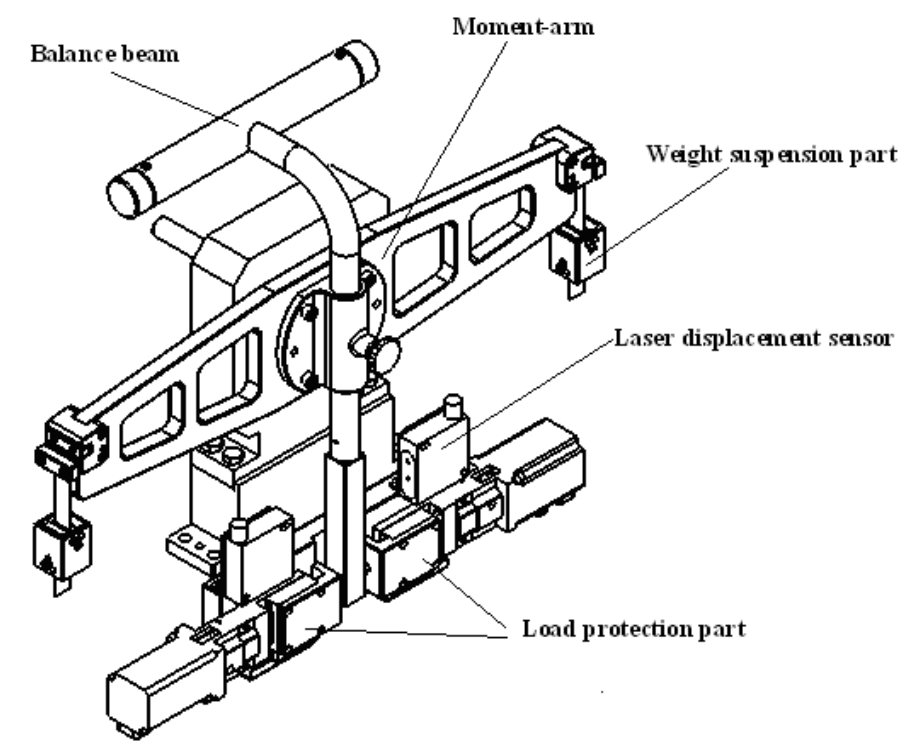

Fig.3. Structure of moment-arm.

\subsection{Weight loading system}

The machine includes two set of weights which are at two sides of moment-arm and may generate clockwise and anticlockwise torque separately in range of $1 \mathrm{mN} \bullet \mathrm{m}-1 \mathrm{~N} \bullet \mathrm{m}$. Each set consists of 5 groups weights: 10 pieces of $0.4 \mathrm{~N}$ weight, 10 pieces of $0.2 \mathrm{~N}$ weight, 10 pieces of $0.08 \mathrm{~N}$ weight, 10 pieces of $0.04 \mathrm{~N}$ weight and 10 pieces of $0.004 \mathrm{~N}$ weight.

For $1 \mathrm{~N} \bullet \mathrm{m}$ torque machine, as the mass of small weights is very light, in order to keep the force applied by small weights accurately and reliably, two special weight loading systems are designed and manufactured. Each weight loading system consists of 
a load frame and a weight support frame. The load frame is like a basket, it has 10 steps on which the working weights are placed. There are 10 layers support paws which are fixed on the support frame, each layer has 5 support paws, 5 groups of weights are placed on the paws of the support frame, the different group of weights may be selected by rotating weight support frame. Before loading, the support frame with groups of weights is moved horizontally to the set position, the selected weights are transported to the load frame from the support frame and torque is applied by lowering or lifting the support frame. The structure of weight loading system is shown in Fig. 4.

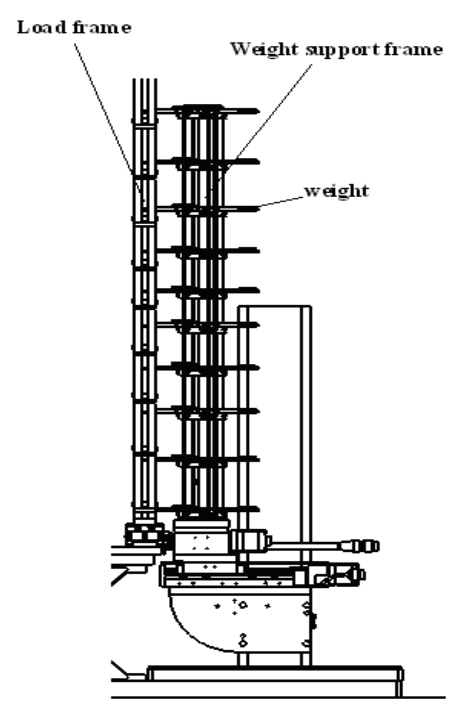

Fig.4. Structure of moment-arm.

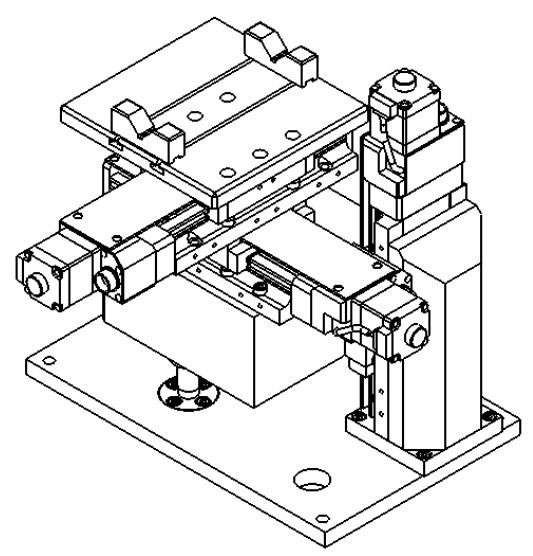

Fig.5. Mounting table.

\subsection{Mounting platform}

For the small torque machine, the rated torque of calibrated torque transducer is very small, during installation, for preventing the damage to the calibrated transducer the parasitic force acting on the transducer has to be avoided. A three-dimensional precision mounting platform is designed which may precisely adjust the position of transducer in $\mathrm{X}, \mathrm{Y}, \mathrm{Z}$ directions and keep alignment of the axis of the transducer and that of torque machine so that transducer is avoided from the impact of parasitic force while mounting. The structure of mounting table is shown in Fig. 5.

\section{Electric Control System}

The industry control computer is used as the controller to realize the centralized control and management of the machine. Programmable controller (PLC) is adopted to control the motions of the execution components, the work statuses of the components are realtime displayed on the screen of computer. The position of the moment-arm is measured by the laser displacement sensors and adjusted accurately by controlling the counter 
bearing drive, so that the moment-arm may be kept in the initial horizontal position after torque applied. The measuring amplifier which is used to measure the output of the torque transducer is communicated with the computer via the serial interface. By designing the complex program, the machine realizes the full automatic control. The block diagram of electrical control is shown in Fig. 6.

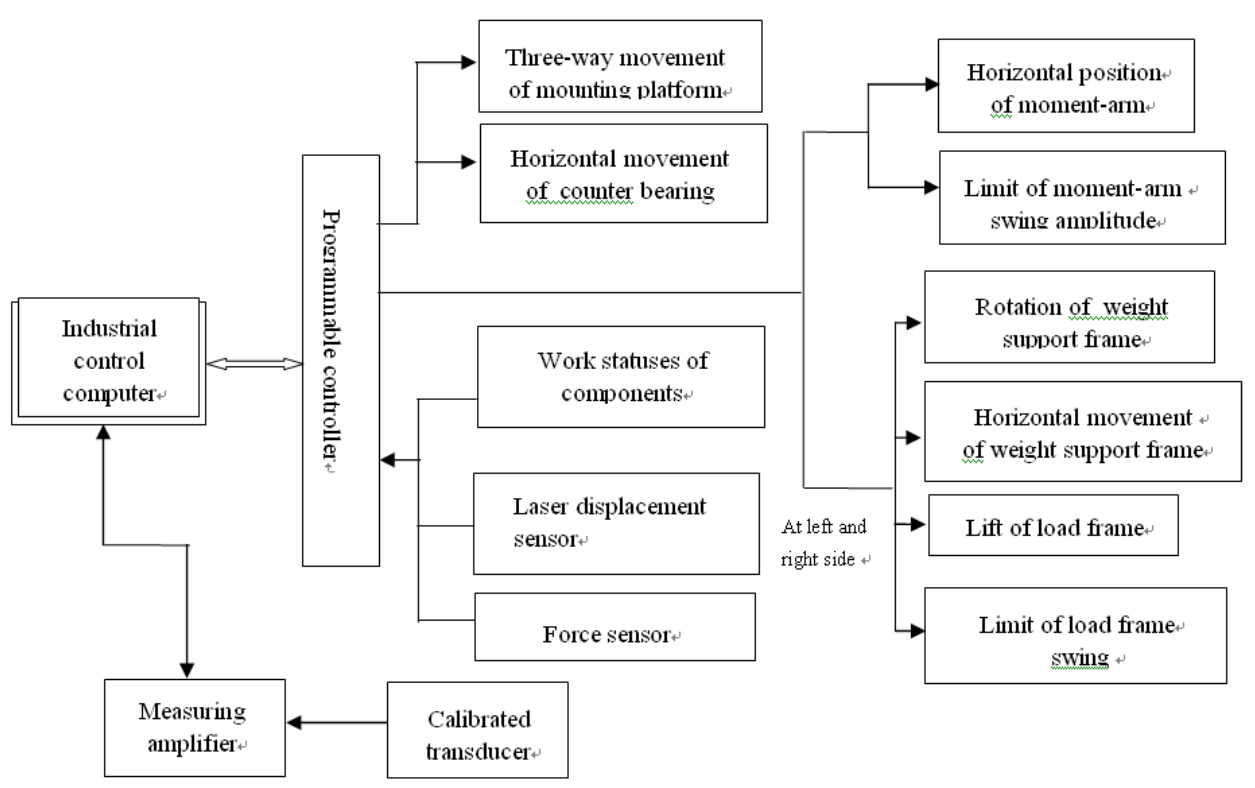

Fig.6. Block diagram of electrical control.

\section{Conclusion and Further Work}

$1 \mathrm{~N} \cdot \mathrm{m}$ torque machine has the following main features: The air bearing with low friction is adopted to support the moment-arm to minimize the friction at the fulcrum and improve the sensitivity of the machine; the invar with low expansion coefficient is used as the material of the moment-arm to reduce the uncertainty caused by the moment-arm length change due to temperature variation; the designed weight suspension system may keep the force generated by small weights vertically downward and be free from the parasitic force; the distinctive weight loading system may ensure the applied force by small weights accurately and reliably and avoid reverse process during loading while calibrating different range of transducers. The torque machine is capable of generating clockwise and anticlockwise torque in range of $1 \mathrm{mN} \cdot \mathrm{m}-1 \mathrm{~N} \cdot \mathrm{m}$, the full automatic control is realized. Up to now the installation and adjustment of the machine have be completed, the performance test is being carried out. The project of establishment of 1 $\mathrm{N} \bullet \mathrm{m}$ torque standard machine will be finished in the end of 2013. 


\section{References}

1. ZHANG Zhimin , LI Tao, ZHANG Yue, and etc., A Full-automatic High Accuracy $100 \mathrm{Nm}$ Torque Standard Machine, IMEKO XIX WORLD CONGRESS, Sept. 6-11, 2009.

2. Atsuhiro NISHINO, Koji OHGUSHI and Kazunaga UEDA, Design and Component Evaluation of the $10 \mathrm{~N} \bullet \mathrm{m}$ Dead-Weight Torque Standard Machine, Proceedings of Asia-Pacific Symposium on Mass, Oct 24 - 252007. 